\title{
A Study on Work Intensity, Work-Life Balance, and Burn- out among Korean Neurosurgeons after the Enactment of the Special Act on Korean Medical Residents
}

\author{
Tae Gon Kim \\ Department of Neurosurgery, CHA Bundang Medical Center, CHA University School of Medicine, Seongnam, Korea
}

Objective : Since the enactment of the Special Act on Korean Medical Residents, neurosurgeons working at training hospitals have been performing the duties of residents, in addition to their existing patient care responsibilities, which include surgery, education, and research. This study explores the relationships between work intensity, work-life balance, and burnout experienced by Korean neurosurgeons.

Methods : The participants $(n=451)$ were neurosurgeons working at training hospitals throughout Republic of Korea. Data on socio-demographic characteristics (including objective and subjective work environment), work intensity, work-life balance, and burnout were gathered using self-report questionnaires completed between March 1 and December 20, 2019. The data were analyzed using descriptive statistics, independent samples t-tests, one-way analysis of variance, Pearson's correlations, and multiple regression analysis. IBM SPSS Statistics for Windows, version 25 (IBM Corp., Armonk, NY, USA) was used for the analyses.

Results : The work intensity, work-life balance, and burnout levels of neurosurgeons were 3.95, 3.57 (on a scale from 1 to 5 ) and 4.60 (on a scale from 1 to 7); and 280 (62.1\%) of 451 neurosurgeons were found to be experiencing burnout. By controlling for the sociodemographic characteristics, the effects of work intensity and work-life balance on burnout were analyzed. Work intensity $(B=0.314)$, work-life balance-family and leisure $(B=0.216)$, work-life balance-growth $(B=0.147)$, job stress $(B=0.133)$, and satisfaction with human relationships $(\mathrm{B}=-0.069)$ were shown to be significant (all $p<0.05)$, and they were found to affect burnout in the abovementioned order. The overall explanatory power was 58.3\% $(p<0.05)$, and the explanatory power with the addition of independent variables such as work intensity and work-life balance was $14.5 \%(p<0.05)$.

Conclusion : This study showed that Korean neurosurgeons working at training hospitals experienced a high level of work intensity and job stress, and low work-life balance. Additionally, nearly half of the neurosurgeons were found to experience burnout related to factors such as work intensity, work-life balance, job stress, and satisfaction with human relationships. In particular, these factors seem to have deteriorated further after the implementation of the Special Act on Korean Medical Residents. These very high levels of burnout among Korean neurosurgeons who care for patients with both brain and spinal diseases may have a very important impact on patients' health. Therefore, it is recommended that the Korean Neurosurgical Society and the Korean government make efforts to improve the factors that affect burnout among Korean neurosurgeons.

Key Words : Neurosurgeons · Work · Work-life balance · Burnout, Psychological · Special act on medical residents.

- Received : August 11, 2020 •Revised : October 21, 2020 •Accepted : October 28, 2020

- Address for reprints : Tae Gon Kim

Department of Neurosurgery, CHA Bundang Medical Center, CHA University School of Medicine, 59 Yatap-ro, Bundang-gu, Seongnam 13496, Korea

Tel : +82-31-780-5260, Fax : +82-31-780-5269, E-mail : tgkim@chamc.co.kr, ORCID : https://orcid.org/0000-0001-6258-6412

This is an Open Access article distributed under the terms of the Creative Commons Attribution Non-Commercial License (http://creativecommons.org/licenses/by-nc/4.0) which permits unrestricted non-commercial use, distribution, and reproduction in any medium, provided the original work is properly cited. 


\section{INTRODUCTION}

On December 23, 2017, the Act on the Improvement of Training Conditions and Status of Medical Residents (also known as the Special Act on Korean Medical Residents) was implemented in the Republic of Korea to improve the training environment and the status of Korean medical residents. This law was enacted to prevent patients from being exposed to the risk of low quality medical services from residents suffering from extreme fatigue due to their heavily demanding duties. Therefore, this law restricts working hours to 80 hours per week to improve the training conditions of the residents, including a 36-hour overtime ban and a minimum of 10 hours of rest after continuous training.

Once this law was implemented, it was expected that the training environment of the residents would be improved; however, hospitals under the Korean Health Insurance, where payment is low (at 75\% of the cost), employ the minimum number of employees and use existing personnel to strengthen labor needs. Therefore, specialists including neurosurgeons working at training hospitals perform the duties of residents; in addition to their existing patient care responsibilities, including surgery, education, and research. Just as the residents' heavy workload increases the risk of low-quality medical services, overburdening specialists can lead to serious deterioration in the quality of medical services.

This study explores the relationships between the current status of the work environment, job stress, work intensity, work-life balance, and burnout among neurosurgeons working at training hospitals. The results of this study can inform future policy to create an environment in which neurosurgeons can provide the best surgical treatment for patients.

\section{MATERIALS AND METHODS}

This study was approved on February 13, 2019 by the Institutional Review Board (IRB) of CHA Bundang Medical Center, CHA University School of Medicine (IRB No. CHAMC 2019-01-036).

\section{Measures and variables}

We conducted a validity survey of the tools selected by our researchers to determine whether the tools are suitable for measuring changes in neurosurgeons' work after the implementation of the Special Act on Korean Medical Residents. The survey included items on sociodemographic characteristics, objective work environment (such as position, hospital size, specialization), subjective work environment (such as satisfaction with salary level), work intensity, work-life balance, and burnout. The experts were 7-10 neurosurgery professors who had been in the training hospital for more than 5 years after the acquisition of a neurosurgery specialty.

\section{Operational definitions}

\section{Work intensity}

Work intensity, based on Janssen's quantitative concept, is defined as the amount of work to be undertaken in large quantities, or the amount of work required to be done faster and harder to handle the same amount of work in a relatively short time ${ }^{5)}$. Janssen approached the concept of work intensity from two perspectives : workload and time. He judged that the shorter the time, the greater the workload and the higher the work intensity. In this study, Janssen's scale to measure the concept of work intensity; time was measured by such items as 'I have to work quickly', while the concept of workload was based on such items as 'I always have the next thing to do even when I finish my current work.' A total of 10 items were used, and the present study was modified and supplemented according to this study ${ }^{5}$. The 5-point Likert scale used to measure the items, and the measurement level of the variables was : 1, not at all; 2, no; 3, normal; 4, yes; and 5, always.

\section{Work-life balance}

Work-life balance generally refers to the harmony between 'work' and 'other than work' that make up life ${ }^{7)}$. In this study, we defined work-life balance as the state that regulates and controls one's life with resources such as time and energy harmoniously distributed between the areas of work and life; and that the individual is satisfied with their perceived balance between work and life. Based on the research of Kim et al. ${ }^{7,8)}$, the study addressed two factors consisting of a total of 16 questions : 10 questions for work-family and leisure and six questions for work-growth. The 5-point Likert scale was used to rate the responses according to 1 , not at all; 2, no; 3 , normal; 4, yes; and 5, always. 


\section{Burnout}

Burnout is defined by lethargy, pessimism, constant complaining, and indifference due to excessive work, study, and activities. In this study, the causes of these responses are limited to job-related stress, and the state experienced by members of the organization due to these stressors are defined as burnout. The Maslach Burnout Inventory (MBI), developed by Maslach and Jackson ${ }^{10)}$, was used to measure burnout. MBI is composed of 22 items consisting of three dimensions such as depersonalization (DP), which shows a negative reaction to the recipient of the service; reduced personal achievement (PA), which is a negative reaction to the achievement of their work; and emotional exhaustion (EE), which is a tired or emotional dry feeling. The MBI is used in most of the studies; Choi and Chung's ${ }^{4)}$ modified measurement tool was used in this study. The measurement scale consist of 22 items in three dimensions : nine items measure EE, five items measure DP, and eight items measure reduced PA. The 7-point Likert frequency scale used to indicate responses to the items and the measurement level of the variables was : 1 , never; 2 , a few times a year or less; 3 , once a month or less; 4 , a few times a month; 5 , once a week; 6 , a few times a week; and 7 , every day.

\section{Online survey using the questionnaires}

The questionnaire consisted of 78 questions including the socio-demographic characteristics (26 questions), working intensity (10 questions), work-life balance (16 questions), and burnout (22 items). The online self-report survey was distributed to all neurosurgery specialists working at the training hospitals registered in the Korean Neurosurgical Society. Participation was voluntary, and the surveys were encoded by assigning code numbers to each document so that the identity of the subjects could not be known. The participants completed self-report questionnaires from March 1 to December 20, 2019.

\section{Statistical analysis}

To explore the relationships between sociodemographic characteristics, work intensity, work-life balance and burnout experienced by neurosurgeons working at training hospitals, the detailed data were analyzed using descriptive statistics, independent samples t-tests, one way analysis of variance, Pearson correlations, and hierarchical multiple regression analysis. The sociodemographic characteristics of the subjects were an- alyzed by frequency, percentage, average, and standard deviation. Correlation analysis and hierarchical multiple regression analysis were conducted for the socio-demographic characteristics, work intensity, work-life balance, and burnout.

\section{RESULTS}

\section{Socio-demographic characteristics and objective work environment (Table 1)}

Of the 885 specialists working at the training hospital, 451 (about 51.0\%) responded to the survey; of these, about 95.1\% (429) were male respondents. The age distribution of respondents was 191 (42.4\%) were 40-49 years old; 136 (30.2\%) 50-59 years old; and 28 (6.2\%) 60 years old or older. Professors comprised the largest group with 72 (38.1\%), and 25 fellows (5.5\%) comprised the smallest group. According to hospital location, 166 doctors (36.8\%) worked in Seoul, and 112 doctors $(24.8 \%)$ worked in Incheon and Gyeonggi-do. The number of doctors working in $800-1000$ bed facilities was the largest with 154 (34.1\%) respondents; the smallest number of doctors work in facilities with less than 500 beds, 62 (13.7\%). According to the type of hospital, 312 doctors (69.2\%) worked at private university hospitals, and 88 doctors (19.5\%) worked at national and public university hospitals. The specializations were divided into the brain and spine, with 285 (63.2\%) working in the brain field and 110 (24.4\%) working in the spine field. There were 150 doctors (33.3\%) who worked in hospitals with more than five residents, but 45 doctors (10\%) worked in hospitals where only one resident worked. In the survey of average working hours per day on weekdays, 174 doctors (38.6\%) responded that they worked 12-16 hours on average, and 163 doctors (36.1\%) responded that they worked 10-12 hours. There were 42 doctors (9.3\%) working for more than 16 hours per day. There were 132 doctors (29.3\%) working twice a month on weekends, and 126 doctors (27.9\%) working more than four times a month. The number of doctors who had 10 days of vacation per year was the highest at 103 (22.8\%), and the number of doctors who had 7 days of vacation was the second highest at 99 (22.0\%). Of these, 138 (30.6\%) were receiving calls more than five times a month from other medical staff other than neurosurgery residents (i.e., nurses or interns), and $76.7 \%$ of the respondents received calls more than once a month. Approximately 57\% of the doctors performed 
Table 1. Socio-demographic characteristics (including objective and subjective work environment)

\begin{tabular}{|c|c|}
\hline & Value \\
\hline \multicolumn{2}{|l|}{ Gender } \\
\hline Male & $429(95.12)$ \\
\hline Female & $22(4.88)$ \\
\hline \multicolumn{2}{|l|}{ Age (years) } \\
\hline $30-39$ & $96(21.29)$ \\
\hline $40-49$ & $191(42.35)$ \\
\hline $50-59$ & $136(30.16)$ \\
\hline$\geq 60$ & $28(6.21)$ \\
\hline \multicolumn{2}{|l|}{ Position } \\
\hline Fellow & $25(5.54)$ \\
\hline Assistanct professor & $109(24.17)$ \\
\hline Associate professor & $98(21.73)$ \\
\hline Professor & $172(38.14)$ \\
\hline Other specialist & $47(10.42)$ \\
\hline \multicolumn{2}{|l|}{ Hospital locations } \\
\hline Seoul & $166(36.81)$ \\
\hline Incheon \& Gyeonggi-do & $112(24.83)$ \\
\hline Gangwon-do & $24(5.32)$ \\
\hline Daejeon \& Chungcheong-do & $40(8.87)$ \\
\hline Jeolla-do & $22(4.88)$ \\
\hline Gyeongsang-do \& Jeju & $87(19.29)$ \\
\hline \multicolumn{2}{|l|}{ Hospital size } \\
\hline Below 500 beds & $62(13.75)$ \\
\hline $500-799$ beds & $110(24.39)$ \\
\hline 800-999 beds & $154(34.15)$ \\
\hline Over 1000 beds & $125(27.72)$ \\
\hline \multicolumn{2}{|l|}{ Hospital type } \\
\hline National \& public university hospital & $88(19.51)$ \\
\hline Private university hospital & $312(69.18)$ \\
\hline National \& public hospital & $25(5.54)$ \\
\hline Private hospital & $26(5.76)$ \\
\hline \multicolumn{2}{|l|}{ Specilization } \\
\hline Brain & $285(63.19)$ \\
\hline Spine & $110(24.39)$ \\
\hline Brain \& spine & $56(12.42)$ \\
\hline \multicolumn{2}{|l|}{ The number of residents } \\
\hline 1 & $45(9.98)$ \\
\hline 2 & $43(9.53)$ \\
\hline 3 & 85 (18.85) \\
\hline 4 & $128(28.38)$ \\
\hline Over 5 & $150(33.26)$ \\
\hline
\end{tabular}

Table 1. Contiuned

\begin{tabular}{lc}
\hline & Value \\
\hline Average working hours per day on weekdays & \\
8 to below 10 hours & $72(15.96)$ \\
10 to below 12 hours & $163(36.14)$ \\
12 to below 16 hours & $174(38.58)$ \\
Over 16 hours & $42(9.31)$ \\
Working days on weekends per month & \\
None & $27(5.99)$ \\
1 & $90(19.96)$ \\
2 & $132(29.27)$ \\
3 & $76(16.85)$ \\
Over 4 & $126(27.94)$ \\
Vacation per year & $8.00 \pm 4.10^{*}$ \\
$0-5$ days & $92(20.40)$ \\
$6-10$ days & $258(57.21)$ \\
$11-15$ days & $84(18.63)$ \\
$16-20$ days & $9(2.00)$ \\
$21-25$ days & $7(1.55)$ \\
26 days & $1(0.22)$
\end{tabular}

\section{Calls from interns or nurses}

$\begin{array}{lc}\text { None } & 105(23.28) \\ 1 & 77(17.07) \\ 2 & 58(12.86) \\ 3 & 30(6.65) \\ 4 & 43(9.53) \\ \text { Over 5 } & 138(30.60)\end{array}$

Surgery without residents

None 194 (43.02)

$1 \quad 51(11.31)$

$2 \quad 52(11.53)$

$3 \quad 30(6.65)$

$444(9.76)$

Over $5 \quad 80(17.74)$

Rounds without residents

None $112(24.83)$

$11118(26.16)$

$2 \quad 68(15.08)$

$3 \quad 54$ (11.97)

$4 \quad 34(7.54)$

Over $5 \quad 65$ (14.41)

Working as usual after overnight surgery

None

$103(22.84)$ 
Table 1. Contiuned

\begin{tabular}{|c|c|}
\hline & Value \\
\hline 1 & $148(32.82)$ \\
\hline 2 & $101(22.39)$ \\
\hline 3 & $61(13.53)$ \\
\hline \multicolumn{2}{|c|}{ The level of job stress } \\
\hline Very low & $5(1.11)$ \\
\hline Low & $15(3.33)$ \\
\hline Normal & $104(23.06)$ \\
\hline High & $178(39.47)$ \\
\hline Very high & $149(33.04)$ \\
\hline \multicolumn{2}{|c|}{ Satisfaction with salary level } \\
\hline Not at all & $179(39.69)$ \\
\hline Not & $116(25.72)$ \\
\hline Normal & $108(23.95)$ \\
\hline Yes & $37(8.20)$ \\
\hline Very yes & $11(2.44)$ \\
\hline \multicolumn{2}{|c|}{ Satisfaction with welfare system } \\
\hline Not at all & $144(31.93)$ \\
\hline Not & $139(30.82)$ \\
\hline Normal & $113(25.06)$ \\
\hline Yes & $40(8.87)$ \\
\hline Very yes & $15(3.33)$ \\
\hline \multicolumn{2}{|c|}{ Satisfaction with human relationships } \\
\hline Not at all & $26(5.76)$ \\
\hline Not & $49(10.86)$ \\
\hline Normal & $173(38.36)$ \\
\hline Yes & $165(36.59)$ \\
\hline Very yes & $38(8.43)$ \\
\hline
\end{tabular}

Values are presented as number (\%). *This value is presented as mean \pm standard deviation

surgery without residents more than once a month, and $73.8 \%$ made rounds without residents more than once a week. A total of $77.2 \%$ of the doctors experienced working-as-usual after overnight surgery more than once a month.

\section{Subjective work environment (Table 1)}

To the question of their level of job stress, 327 (72.5\%) respondents answered "high" or "very high." Regarding their satisfaction with their salary level, 295 (65.4\%) respondents answered that they were "not at all" or "not" satisfied. Similarly, 283 (62.7\%) respondents answered "not at all” or "not" to the question of their satisfaction with the welfare system. However, 376 (83.4\%) respondents answered "normal," "yes," or "very yes" to the question about satisfaction with human relationships.

\section{Special questions related to the Special Act on Korean Medical Residents}

Five questions were added to understand the situation related to the Special Act on Korean Medical Residents (Table 2). These questions were made to gain an understanding of the situations, but they were not scientifically validated tools. Respondents were asked "If I'm given more time, I'll be able to concentrate more on patient care," 269 (59.6\%) respondents answered "yes" or "very yes" to the question. To the question "After the Special Act on Korean Medical Residents, the satisfaction of neurosurgical patients was lower than before," 278 $(61.6 \%)$ of respondents answered "yes" or "very yes." To the question "I am very busy because I often have to give a level of explanation that my residents can do," 318 (70.5\%) respondents answered "yes" or "very yes." When asked whether "Normal patient care is difficult without assistant personnel such as physician assistants or nurse practitioners," 399 $(88.5 \%)$ respondents answered "yes" or "very yes." Finally, 383 $(84.9 \%)$ respondents answered "yes" or "very yes" to the question "If I'm given more time, I'll be able to do more research and attend conferences."

\section{Validity analysis of the variables}

The validity of the variables was analyzed by factor analysis using the Varimax rotation method. Factors with eigenvalues greater than 1.0 were extracted, and items with factor loadings of 0.50 or greater were extracted. Before conducting the factor analysis, Kaiser-Meyer-Olkin (KMO) indices were used to determine whether the number of items and the sample size were sufficient for using factor analysis, and Bartlett's sphericity test was conducted for the identity matrix test.

The KMO index for work intensity was 0.943 , indicating that the number of items and the sample size are appropriate for the factor analysis, and Bartlett's sphericity test showed that it was not an identity matrix $(p<0.001)$. The factor loadings derived from the factor analysis on work intensity ranged in value from 0.617 to -0.888 , which were greater than 0.50 ; and one factor was extracted which explained $65.45 \%$ of the total variance. 
Table 2. Special questions related to the Special Act on Korean Medical Residents

\begin{tabular}{|c|c|}
\hline & Value \\
\hline \multicolumn{2}{|l|}{ Q1 } \\
\hline Not at all & 17 (3.8) \\
\hline Not & $35(7.8)$ \\
\hline Normal & $130(28.8)$ \\
\hline Yes & $126(27.9)$ \\
\hline Very yes & $143(31.7)$ \\
\hline \multicolumn{2}{|l|}{ Q2 } \\
\hline Not at all & $17(3.8)$ \\
\hline Not & $43(9.5)$ \\
\hline Normal & $113(25.1)$ \\
\hline Yes & $129(28.6)$ \\
\hline Very yes & $149(33.0)$ \\
\hline \multicolumn{2}{|l|}{ Q3 } \\
\hline Not at all & $8(1.8)$ \\
\hline Not & $37(8.2)$ \\
\hline Normal & 88 (19.5) \\
\hline Yes & $166(36.8)$ \\
\hline Very yes & $152(33.7)$ \\
\hline \multicolumn{2}{|l|}{ Q4 } \\
\hline Not at all & $6(1.3)$ \\
\hline Not & $12(2.7)$ \\
\hline Normal & $34(7.5)$ \\
\hline Yes & 88 (19.5) \\
\hline Very yes & $311(69.0)$ \\
\hline \multicolumn{2}{|l|}{ Q5 } \\
\hline Not at all & $5(1.1)$ \\
\hline Not & $11(2.4)$ \\
\hline Normal & $52(11.5)$ \\
\hline Yes & 157 (34.8) \\
\hline Very yes & $226(50.1)$ \\
\hline
\end{tabular}

Values are presented as number (\%). Q1. If I'm given more time, I'll be able to concentrate more on patient care. Q2. After the Special Act on Korean Medical Residents, the satisfaction of neurosurgical patients was lower than before. Q3. I am busy and busy because I often have to explain the level of explanation that my residents can do. Q4. Normal patient care is difficult without assistant personnel such as Physician assistants or nurse practitioners. Q5. If I'm given more time, I'll be able to do more research and attend conferences

The KMO index for work-life balance was 0.932 indicating that the data were appropriate for the factor analysis, and Bartlett's sphericity test showed that it was not an identity ma- trix $(p<0.001)$. Therefore, these data were appropriate for conducting the factor analysis. The factor analysis resulted in a two-factor solution; the factors were named as 'work-life balance-family and leisure,' and 'work-life balance-growth,' and the factor loadings ranged in value from 0.469 to -0.801 , which were greater than 0.50 with the exception of one question. The two factors accounted for $61.28 \%$ of the total variance.

The KMO index for burnout was 0.922 , appropriate for the factor analysis, and Bartlett's sphericity test showed that it was not an identity matrix $(p<0.001)$. The results of the factor analysis on burnout confirmed the three-factor solution. The factor names were consistent with previous research : 'emotional exhaustion,' 'depersonalization,' and 'reduced personal achievement.' The factor loadings ranged in value from 0.522 to -0.876 , which were greater than 0.50 . The three factors accounted for $59.15 \%$ of the total variance.

\section{Reliability analysis \& normality test}

Reliability analysis was conducted using Cronbach's alpha to measure the internal consistency of the items. Internal consistency is deemed "reliable" if it is 0.70 or higher, and "acceptable" if it is greater than $0.60^{12)}$. The reliability of work intensity is 0.935 , work-life balance is 0.936 , and burnout is 0.863 ; thus, all scales are above 0.70 and all reliable.

The normality test of the scale was based on skewness and kurtosis, and the normal distribution was assumed if the absolute value of the skewness and kurtosis was less than $3.0^{9}$. The degree of skewness and kurtosis of the tools used in this study were all below 3.0 indicating that they were normally distributed.

\section{Analysis of the relationships between the socio- demographic characteristics and work intensity, work-life balance, and burnout}

\section{Work intensity}

The relationship between work intensity and socio-demographic characteristics is described in detail in Table 3. Generally, the derived results were consistent with general expectations. The work intensity was higher in respondents under 60 , higher for the associate professors than other specialists. In the case of hospitals with more beds, many residents, greater average number of working hours per day on weekdays, greater number of working days on weekends per month, and fewer 
J Korean Neurosurg Soc 64 | July 2021

Table 3. Work intensity according to socio-demographic characteristics

\begin{tabular}{|c|c|c|c|c|c|}
\hline & Value & Mean \pm SD & Post hoc & $t / F$ & $p$-value \\
\hline \multicolumn{6}{|l|}{ Gender } \\
\hline Male & 429 (95.12) & $3.95 \pm 0.70$ & & -0.682 & 0.496 \\
\hline Female & $22(4.88)$ & $4.05 \pm 0.79$ & & & \\
\hline Age (years) & & & $b, c, a>d$ & 9.261 & $<0.001$ \\
\hline 30-39 (a) & $96(21.29)$ & $3.88 \pm 0.74$ & & & \\
\hline $40-49$ (b) & $191(42.35)$ & $4.10 \pm 0.62$ & & & \\
\hline $50-59$ (c) & $136(30.16)$ & $3.90 \pm 0.70$ & & & \\
\hline$\geq 60$ (d) & $28(6.21)$ & $3.41 \pm 0.76$ & & & \\
\hline Position & & & $c>e$ & 2.867 & 0.023 \\
\hline Fellow (a) & $25(5.54)$ & $3.91 \pm 0.74$ & & & \\
\hline Assistanct professor (b) & $109(24.17)$ & $3.99 \pm 0.70$ & & & \\
\hline Associate professor (c) & $98(21.73)$ & $4.13 \pm 0.61$ & & & \\
\hline Professor (d) & $172(38.14)$ & $3.88 \pm 0.74$ & & & \\
\hline Other specialist (e) & $47(10.42)$ & $3.79 \pm 0.64$ & & & \\
\hline Hospital locations & & & & 2.082 & 0.067 \\
\hline Seoul & $166(36.81)$ & $4.06 \pm 0.72$ & & & \\
\hline Incheon \& Gyeonggi-do & $112(24.83)$ & $3.87 \pm 0.77$ & & & \\
\hline Gangwon-do & $24(5.32)$ & $4.12 \pm 0.59$ & & & \\
\hline Daejeon \& Chungcheong-do & $40(8.87)$ & $3.86 \pm 0.75$ & & & \\
\hline Jeolla-do & $22(4.88)$ & $4.00 \pm 0.45$ & & & \\
\hline Gyeongsang-do \& Jeju & $87(19.29)$ & $3.83 \pm 0.61$ & & & \\
\hline Hospital size & & & $d>b$ & 3.937 & 0.009 \\
\hline Below 500 beds (a) & $62(13.75)$ & $3.91 \pm 0.67$ & & & \\
\hline 500-799 beds (b) & $110(24.39)$ & $3.85 \pm 0.67$ & & & \\
\hline 800-999 beds (c) & $154(34.15)$ & $3.89 \pm 0.70$ & & & \\
\hline Over 1000 beds (d) & $125(27.72)$ & $4.13 \pm 0.72$ & & & \\
\hline Hospital type & & & & 1.998 & 0.114 \\
\hline National \& public university hospital & $88(19.51)$ & $3.98 \pm 0.76$ & & & \\
\hline Private university hospital & $312(69.18)$ & $3.98 \pm 0.69$ & & & \\
\hline National \& public hospital & $25(5.54)$ & $3.83 \pm 0.65$ & & & \\
\hline Private hospital & $26(5.76)$ & $3.65 \pm 0.62$ & & & \\
\hline Specilization & & & & 1.233 & 0.292 \\
\hline Brain & $285(63.19)$ & $3.92 \pm 0.73$ & & & \\
\hline Spine & $110(24.39)$ & $3.95 \pm 0.69$ & & & \\
\hline Brain \& spine & $56(12.42)$ & $4.09 \pm 0.57$ & & & \\
\hline The number of residents & & & $e>b$ & 7.714 & $<0.001$ \\
\hline 1 (a) & 45 (9.98) & $3.94 \pm 0.64$ & & & \\
\hline 2 (b) & $43(9.53)$ & $3.70 \pm 0.80$ & & & \\
\hline $3(c)$ & $85(18.85)$ & $3.81 \pm 0.69$ & & & \\
\hline $4(d)$ & $128(28.38)$ & $3.85 \pm 0.69$ & & & \\
\hline Over 5 (e) & $150(33.26)$ & $4.19 \pm 0.64$ & & & \\
\hline
\end{tabular}


Burnout of Korean Neurosurgeons | Kim TG

Table 3. Contiuned

\begin{tabular}{|c|c|c|c|c|c|}
\hline & Value & Mean \pm SD & Post hoc & $t / F$ & $p$-value \\
\hline Average working hours per day on weekdays & & & $d, c>b>a$ & 63.033 & $<0.001$ \\
\hline 8 to below 10 hours (a) & $72(15.96)$ & $3.27 \pm 0.64$ & & & \\
\hline 10 to below 12 hours (b) & $163(36.14)$ & $3.78 \pm 0.57$ & & & \\
\hline 12 to below 16 hours (c) & $174(38.58)$ & $4.28 \pm 0.53$ & & & \\
\hline Over 16 hours (d) & $42(9.31)$ & $4.42 \pm 0.80$ & & & \\
\hline Working days on weekends per month & & & $e>b>a$ & 16.227 & $<0.001$ \\
\hline None (a) & $27(5.99)$ & $3.21 \pm 0.79$ & & & \\
\hline 1 (b) & $90(19.96)$ & $3.80 \pm 0.63$ & & & \\
\hline $2(c)$ & $132(29.27)$ & $3.87 \pm 0.71$ & & & \\
\hline $3(d)$ & $76(16.85)$ & $4.09 \pm 0.61$ & & & \\
\hline Over 4 (e) & $126(27.94)$ & $4.22 \pm 0.62$ & & & \\
\hline Vacation per year & & & $a, b>e$ & 4.648 & 0.005 \\
\hline$<5$ days (a) & $92(20.40)$ & $4.11 \pm 0.61$ & & & \\
\hline $6-10$ days (b) & $258(57.21)$ & $4.00 \pm 0.64$ & & & \\
\hline 11-15 days (c) & 84 (18.63) & $3.69 \pm 0.84$ & & & \\
\hline $16-20$ days $(\mathrm{d})$ & $9(2.00)$ & $3.82 \pm 0.72$ & & & \\
\hline$>21$ days (e) & $8(1.77)$ & $3.21 \pm 0.99$ & & & \\
\hline Calls from interns or nurses & & & $d>e$ & 2.628 & 0.026 \\
\hline None (a) & $105(23.28)$ & $3.84 \pm 0.80$ & & & \\
\hline 1 (b) & 77 (17.07) & $3.91 \pm 0.63$ & & & \\
\hline $2(c)$ & $58(12.86)$ & $3.88 \pm 0.77$ & & & \\
\hline $3(d)$ & $30(6.65)$ & $4.11 \pm 0.66$ & & & \\
\hline 4 (e) & $43(9.53)$ & $3.82 \pm 0.62$ & & & \\
\hline Over 5 (f) & $138(30.60)$ & $4.09 \pm 0.63$ & & & \\
\hline Surgery without residents & & & $f>a$ & 3.768 & 0.002 \\
\hline None (a) & $194(43.02)$ & $3.82 \pm 0.75$ & & & \\
\hline $1(b)$ & 51 (11.31) & $3.97 \pm 0.66$ & & & \\
\hline $2(c)$ & $52(11.53)$ & $4.04 \pm 0.63$ & & & \\
\hline $3(d)$ & $30(6.65)$ & $3.98 \pm 0.68$ & & & \\
\hline 4 (e) & $44(9.76)$ & $3.93 \pm 0.69$ & & & \\
\hline Over 5 (f) & $80(17.74)$ & $4.20 \pm 0.58$ & & & \\
\hline Rounds without residents & & & & 1.274 & 0.274 \\
\hline None & $112(24.83)$ & $3.86 \pm 0.78$ & & & \\
\hline 1 & $118(26.16)$ & $3.90 \pm 0.66$ & & & \\
\hline 2 & $68(15.08)$ & $4.06 \pm 0.74$ & & & \\
\hline 3 & $54(11.97)$ & $3.94 \pm 0.65$ & & & \\
\hline 4 & $34(7.54)$ & $4.03 \pm 0.53$ & & & \\
\hline Over 5 & 65 (14.41) & $4.05 \pm 0.71$ & & & \\
\hline Working as usual after overnight surgery & & & $e>b>a$ & 14.411 & $<0.001$ \\
\hline None (a) & $103(22.84)$ & $3.64 \pm 0.78$ & & & \\
\hline $1(b)$ & $148(32.82)$ & $3.89 \pm 0.67$ & & & \\
\hline
\end{tabular}


Table 3. Contiuned

\begin{tabular}{|c|c|c|c|c|c|}
\hline & Value & Mean \pm SD & Post hoc & $t / F$ & $p$-value \\
\hline $2(c)$ & $101(22.39)$ & $4.05 \pm 0.68$ & & & \\
\hline $3(d)$ & $61(13.53)$ & $4.23 \pm 0.52$ & & & \\
\hline Over 4 (e) & $38(8.43)$ & $4.35 \pm 0.48$ & & & \\
\hline The level of job stress & & & $\begin{array}{c}e>c>a \\
d>b\end{array}$ & 38.005 & $<0.001$ \\
\hline Not at all (a) & $5(1.11)$ & $2.66 \pm 1.40$ & & & \\
\hline Not (b) & $15(3.33)$ & $2.98 \pm 0.70$ & & & \\
\hline Normal (c) & $104(23.06)$ & $3.51 \pm 0.61$ & & & \\
\hline Yes (d) & $178(39.47)$ & $3.97 \pm 0.53$ & & & \\
\hline Very yes (e) & $149(33.04)$ & $4.37 \pm 0.58$ & & & \\
\hline \multicolumn{6}{|l|}{ Satisfaction with salary level } \\
\hline Not at all (a) & 179 (39.69) & $4.09 \pm 0.66$ & $e>c$ & 3.444 & 0.009 \\
\hline Not (b) & $116(25.72)$ & $3.84 \pm 0.67$ & & & \\
\hline Normal (c) & $108(23.95)$ & $3.83 \pm 0.78$ & & & \\
\hline Yes (d) & $37(8.20)$ & $3.94 \pm 0.64$ & & & \\
\hline Very yes (e) & $11(2.44)$ & $4.11 \pm 0.72$ & & & \\
\hline Satisfaction with welfare system & & & & 2.217 & 0.066 \\
\hline Not at all & 144 (31.93) & $4.08 \pm 0.60$ & & & \\
\hline Not & 139 (30.82) & $3.86 \pm 0.70$ & & & \\
\hline Normal & $113(25.06)$ & $3.88 \pm 0.78$ & & & \\
\hline Yes & $40(8.87)$ & $3.98 \pm 0.66$ & & & \\
\hline Very yes & $15(3.33)$ & $3.98 \pm 0.87$ & & & \\
\hline \multicolumn{6}{|c|}{ Satisfaction with human relationships } \\
\hline Not at all & $26(5.76)$ & $4.24 \pm 0.66$ & & 1.813 & 0.125 \\
\hline Not & $49(10.86)$ & $3.96 \pm 0.77$ & & & \\
\hline Normal & 173 (38.36) & $3.87 \pm 0.75$ & & & \\
\hline Yes & 165 (36.59) & $3.98 \pm 0.62$ & & & \\
\hline Very yes & $38(8.43)$ & $4.00 \pm 0.70$ & & & \\
\hline
\end{tabular}

Values are presented as number (\%). SD : standard deviation

vacation days per year, the work intensity was higher. The higher the frequency of surgery being performed without residents, and of work being done as-usual after the overnight surgery, the higher the work intensity. The work intensity was higher for respondents with high levels of job stress and high satisfaction with salary level, but satisfaction with the welfare system was not related to work intensity. In addition, gender, hospital location, hospital type, specialization, rounds without residents, and satisfaction with human relationships were not related to work intensity. The work intensity in the case of direct calls from interns or nurses three times a month was greater than in the case of four times a month, which is not consistent with the general expectation.

\section{Work-life balance}

The relationship between work-life balance and the sociodemographic characteristics is described in detail in Table 4. The results were consistent with general expectations. Female, respondents in the 30-40 age group, fellows, and those with both brain and spine specializations reported low levels of work-life balance. Respondents who reported working under any of the following work-related situations also experienced 
Burnout of Korean Neurosurgeons | Kim TG

Table 4. Work-life balance according to socio-demographic characteristics

\begin{tabular}{|c|c|c|c|c|c|}
\hline & Value & Mean \pm SD & Post hoc & $t / F$ & $p$-value \\
\hline Gender & & & & -3.223 & 0.001 \\
\hline Male & 429 (95.12) & $3.54 \pm 0.72$ & & & \\
\hline Female & $22(4.88)$ & $4.05 \pm 0.75$ & & & \\
\hline Age (years) & & & $a, b>d$ & 10.366 & $<0.001$ \\
\hline $30-39$ (a) & $96(21.29)$ & $3.73 \pm 0.65$ & & & \\
\hline $40-49(b)$ & $191(42.35)$ & $3.68 \pm 0.71$ & & & \\
\hline $50-59$ (c) & $136(30.16)$ & $3.39 \pm 0.74$ & & & \\
\hline$\geq 60$ (d) & $28(6.21)$ & $3.10 \pm 0.74$ & & & \\
\hline Position & & & $a>d$ & 4.081 & 0.003 \\
\hline Fellow (a) & $25(5.54)$ & $3.82 \pm 0.69$ & & & \\
\hline Assistanct professor (b) & 109 (24.17) & $3.70 \pm 0.68$ & & & \\
\hline Associate professor (c) & $98(21.73)$ & $3.65 \pm 0.68$ & & & \\
\hline Professor (d) & $172(38.14)$ & $3.41 \pm 0.76$ & & & \\
\hline Other specialist (e) & $47(10.42)$ & $3.59 \pm 0.75$ & & & \\
\hline Hospital locations & & & & 0.609 & 0.693 \\
\hline Seoul & $166(36.81)$ & $3.55 \pm 0.70$ & & & \\
\hline Incheon \& Gyeonggi-do & $112(24.83)$ & $3.54 \pm 0.79$ & & & \\
\hline Gangwon-do & $24(5.32)$ & $3.76 \pm 0.70$ & & & \\
\hline Daejeon \& Chungcheong-do & $40(8.87)$ & $3.54 \pm 0.71$ & & & \\
\hline Jeolla-do & $22(4.88)$ & $3.74 \pm 0.67$ & & & \\
\hline Gyeongsang-do \& Jeju & $87(19.29)$ & $3.55 \pm 0.75$ & & & \\
\hline Hospital size & & & & 1.832 & 0.140 \\
\hline Below 500 beds & $62(13.75)$ & $3.43 \pm 0.76$ & & & \\
\hline $500-799$ beds & $110(24.39)$ & $3.62 \pm 0.70$ & & & \\
\hline 800-999 beds & $154(34.15)$ & $3.51 \pm 0.74$ & & & \\
\hline Over 1000 beds & 125 (27.72) & $3.66 \pm 0.73$ & & & \\
\hline Hospital type & & & & 2.150 & 0.093 \\
\hline National \& public university hospital & $88(19.51)$ & $3.74 \pm 0.71$ & & & \\
\hline Private university hospital & 312 (69.18) & $3.54 \pm 0.72$ & & & \\
\hline National \& public hospital & $25(5.54)$ & $3.41 \pm 0.76$ & & & \\
\hline Private hospital & $26(5.76)$ & $3.56 \pm 0.86$ & & & \\
\hline Specilization & & & $c>b$ & 4.120 & 0.017 \\
\hline Brain (a) & 285 (63.19) & $3.62 \pm 0.76$ & & & \\
\hline Spine (b) & $110(24.39)$ & $3.40 \pm 0.65$ & & & \\
\hline Brain \& spine (c) & $56(12.42)$ & $3.64 \pm 0.68$ & & & \\
\hline The number of residents & & & $e>b$ & 2.710 & 0.030 \\
\hline 1 (a) & $45(9.98)$ & $3.54 \pm 0.70$ & & & \\
\hline 2 (b) & $43(9.53)$ & $3.40 \pm 0.80$ & & & \\
\hline $3(c)$ & $85(18.85)$ & $3.61 \pm 0.74$ & & & \\
\hline $4(d)$ & $128(28.38)$ & $3.46 \pm 0.75$ & & & \\
\hline Over 5 (e) & $150(33.26)$ & $3.70 \pm 0.68$ & & & \\
\hline
\end{tabular}


Table 4. Contiuned

\begin{tabular}{|c|c|c|c|c|c|}
\hline & Value & Mean \pm SD & Post hoc & $t / F$ & $p$-value \\
\hline Average working hours per day on weekdays & & & $d, c>b>a$ & 35.713 & $<0.001$ \\
\hline 8 to below 10 hours (a) & $72(15.96)$ & $3.00 \pm 0.66$ & & & \\
\hline 10 to below 12 hours (b) & $163(36.14)$ & $3.43 \pm 0.67$ & & & \\
\hline 12 to below 16 hours (c) & $174(38.58)$ & $3.83 \pm 0.63$ & & & \\
\hline Over 16 hours (d) & $42(9.31)$ & $4.00 \pm 0.73$ & & & \\
\hline Working days on weekends per month & & & $e, d>b>a$ & 17.034 & $<0.001$ \\
\hline None (a) & $27(5.99)$ & $2.85 \pm 0.65$ & & & \\
\hline $1(b)$ & 90 (19.96) & $3.28 \pm 0.70$ & & & \\
\hline $2(c)$ & $132(29.27)$ & $3.57 \pm 0.64$ & & & \\
\hline $3(d)$ & $76(16.85)$ & $3.76 \pm 0.72$ & & & \\
\hline Over 4 (e) & $126(27.94)$ & $3.82 \pm 0.70$ & & & \\
\hline Vacation per year & & & $a>e$ & 5.458 & $<0.001$ \\
\hline$<5$ days (a) & $92(20.40)$ & $3.75 \pm 0.70$ & & & \\
\hline 6-10 days (b) & $258(57.21)$ & $3.60 \pm 0.70$ & & & \\
\hline $11-15$ days (c) & $84(18.63)$ & $3.34 \pm 0.73$ & & & \\
\hline 16-20 days (d) & $9(2.00)$ & $3.40 \pm 0.87$ & & & \\
\hline$>21$ days (e) & $7(1.55)$ & $2.95 \pm 1.03$ & & & \\
\hline Calls from interns or nurses & & & $d>a$ & 5.914 & $<0.001$ \\
\hline None (a) & $105(23.28)$ & $3.33 \pm 0.71$ & & & \\
\hline 1 (b) & $77(17.07)$ & $3.47 \pm 0.70$ & & & \\
\hline 2 (c) & $58(12.86)$ & $3.67 \pm 0.70$ & & & \\
\hline $3(d)$ & $30(6.65)$ & $3.82 \pm 0.66$ & & & \\
\hline $4(\mathrm{e})$ & $43(9.53)$ & $3.43 \pm 0.76$ & & & \\
\hline Over 5 (f) & $138(30.60)$ & $3.76 \pm 0.72$ & & & \\
\hline Surgery without residents & & & $f>a$ & 5.803 & $<0.001$ \\
\hline None (a) & $194(43.02)$ & $3.38 \pm 0.76$ & & & \\
\hline 1 (b) & $51(11.31)$ & $3.58 \pm 0.76$ & & & \\
\hline 2 (c) & $52(11.53)$ & $3.73 \pm 0.63$ & & & \\
\hline $3(d)$ & $30(6.65)$ & $3.68 \pm 0.57$ & & & \\
\hline $4(\mathrm{e})$ & $44(9.76)$ & $3.63 \pm 0.67$ & & & \\
\hline Over 5 (f) & $80(17.74)$ & $3.84 \pm 0.68$ & & & \\
\hline \multicolumn{6}{|l|}{ Rounds without residents } \\
\hline None (a) & $112(24.83)$ & $3.39 \pm 0.78$ & $f>a$ & 3.073 & 0.010 \\
\hline $1(b)$ & $118(26.16)$ & $3.54 \pm 0.65$ & & & \\
\hline 2 (c) & $68(15.08)$ & $3.66 \pm 0.74$ & & & \\
\hline $3(d)$ & $54(11.97)$ & $3.56 \pm 0.67$ & & & \\
\hline 4 (e) & $34(7.54)$ & $3.73 \pm 0.73$ & & & \\
\hline Over 5 (f) & $65(14.41)$ & $3.77 \pm 0.75$ & & & \\
\hline Working as usual after overnight surgery & & & $\begin{array}{c}e, d>b \\
c>a\end{array}$ & 20.844 & $<0.001$ \\
\hline None (a) & $103(22.84)$ & $3.18 \pm 0.70$ & & & \\
\hline
\end{tabular}


Table 4. Contiuned

\begin{tabular}{|c|c|c|c|c|c|}
\hline & Value & Mean \pm SD & Post hoc & $t / F$ & $p$-value \\
\hline 1 (b) & $148(32.82)$ & $3.44 \pm 0.63$ & & & \\
\hline $2(c)$ & $101(22.39)$ & $3.78 \pm 0.72$ & & & \\
\hline $3(d)$ & $61(13.53)$ & $3.85 \pm 0.60$ & & & \\
\hline Over 4 (e) & $38(8.43)$ & $4.11 \pm 0.77$ & & & \\
\hline The level of job stress & & & $\mathrm{e}, \mathrm{d}>\mathrm{c}>\mathrm{a}$ & 111.63 & $<0.001$ \\
\hline Not at all (a) & $5(1.11)$ & $2.20 \pm 0.19$ & & & \\
\hline Not (b) & $15(3.33)$ & $2.58 \pm 0.52$ & & & \\
\hline Normal (c) & $104(23.06)$ & $3.00 \pm 0.58$ & & & \\
\hline Yes (d) & 178 (39.47) & $3.60 \pm 0.51$ & & & \\
\hline Very yes (e) & 149 (33.04) & $4.07 \pm 0.64$ & & & \\
\hline \multicolumn{6}{|l|}{ Satisfaction with salary level } \\
\hline Not at all (a) & 179 (39.69) & $3.81 \pm 0.71$ & $a>e$ & 9.201 & $<0.001$ \\
\hline Not (b) & $116(25.72)$ & $3.49 \pm 0.67$ & & & \\
\hline Normal (c) & $108(23.95)$ & $3.35 \pm 0.73$ & & & \\
\hline Yes (d) & $37(8.20)$ & $3.41 \pm 0.70$ & & & \\
\hline Very yes (e) & $11(2.44)$ & $3.28 \pm 0.74$ & & & \\
\hline Satisfaction with welfare system & & & $a>d$ & 5.803 & $<0.001$ \\
\hline Not at all (a) & 144 (31.93) & $3.81 \pm 0.72$ & & & \\
\hline Not (b) & 139 (30.82) & $3.46 \pm 0.71$ & & & \\
\hline Normal (c) & $113(25.06)$ & $3.47 \pm 0.70$ & & & \\
\hline Yes (d) & $40(8.87)$ & $3.42 \pm 0.70$ & & & \\
\hline Very yes (e) & $15(3.33)$ & $3.44 \pm 0.84$ & & & \\
\hline Satisfaction with human relationships & & & $\begin{array}{c}a>c \\
b>e, d\end{array}$ & 8.097 & $<0.001$ \\
\hline Not at all (a) & $26(5.76)$ & $4.05 \pm 0.65$ & & & \\
\hline Not (b) & $49(10.86)$ & $3.89 \pm 0.77$ & & & \\
\hline Normal (c) & $173(38.36)$ & $3.59 \pm 0.73$ & & & \\
\hline Yes (d) & $165(36.59)$ & $3.41 \pm 0.67$ & & & \\
\hline Very yes (e) & $38(8.43)$ & $3.41 \pm 0.76$ & & & \\
\hline
\end{tabular}

Values are presented as number (\%). SD : standard deviation

poor work-life balance : having a large number of residents, long working hours per day on weekdays, long working days on weekends per month, few vacation days per year; frequent calls from interns or nurses, surgeries without residents, or rounds without residents, frequent days of working-as-usual after overnight surgery; as well as respondents who indicated that they experienced a high level of job stress, low-level satisfaction with salary level, low-level satisfaction with welfare, or low-level satisfaction with human relationships. Hospital location, hospital size, and hospital type were not related to work- life balance.

\section{Burnout}

The relationship between burnout and the socio-demographic characteristics, which is consistent with general expectations, is described in detail in Table 5. Respondents in the 30-40 age group indicated significant burnout. Respondents indicating that they work with a large number of residents, have long working hours per day on weekdays, long working days on weekends per month, few vacation days per 
J Korean Neurosurg Soc 64 | July 2021

Table 5. Burnout according to socio-demographic characteristics

\begin{tabular}{|c|c|c|c|c|c|}
\hline & Value & Mean $\pm S D$ & Post hoc & $t / F$ & $p$-value \\
\hline Gender & & & & -2.438 & 0.015 \\
\hline Male & 429 (95.12) & $4.58 \pm 0.72$ & & & \\
\hline Female & $22(4.88)$ & $4.97 \pm 0.82$ & & & \\
\hline Age (years) & & & $b, a>d$ & 4.454 & 0.004 \\
\hline 30-39 (a) & $96(21.29)$ & $4.66 \pm 0.70$ & & & \\
\hline $40-49$ (b) & $191(42.35)$ & $4.68 \pm 0.72$ & & & \\
\hline $50-59$ (c) & $136(30.16)$ & $4.52 \pm 0.70$ & & & \\
\hline$\geq 60$ (d) & $28(6.21)$ & $4.21 \pm 0.85$ & & & \\
\hline Position & & & & 1.379 & 0.240 \\
\hline Fellow & $25(5.54)$ & $4.71 \pm 0.73$ & & & \\
\hline Assistanct professor & $109(24.17)$ & $4.68 \pm 0.70$ & & & \\
\hline Associate professor & $98(21.73)$ & $4.66 \pm 0.70$ & & & \\
\hline Professor & $172(38.14)$ & $4.50 \pm 0.73$ & & & \\
\hline Other specialist & $47(10.42)$ & $4.60 \pm 0.80$ & & & \\
\hline Hospital locations & & & & 1.012 & 0.410 \\
\hline Seoul & 166 (36.81) & $4.67 \pm 0.72$ & & & \\
\hline Incheon \& Gyeonggi-do & $112(24.83)$ & $4.58 \pm 0.71$ & & & \\
\hline Gangwon-do & $24(5.32)$ & $4.68 \pm 0.83$ & & & \\
\hline Daejeon \& Chungcheong-do & 40 (8.87) & $4.56 \pm 0.77$ & & & \\
\hline Jeolla-do & $22(4.88)$ & $4.66 \pm 0.49$ & & & \\
\hline Gyeongsang-do \& Jeju & $87(19.29)$ & $4.47 \pm 0.75$ & & & \\
\hline Hospital size & & & & 1.092 & 0.352 \\
\hline Below 500 beds & $62(13.75)$ & $4.56 \pm 0.68$ & & & \\
\hline $500-799$ beds & $110(24.39)$ & $4.64 \pm 0.75$ & & & \\
\hline 800-999 beds & $154(34.15)$ & $4.53 \pm 0.72$ & & & \\
\hline Over 1000 beds & 125 (27.72) & $4.68 \pm 0.73$ & & & \\
\hline Hospital type & & & & 0.380 & 0.767 \\
\hline National \& public university hospital & $88(19.51)$ & $4.66 \pm 0.76$ & & & \\
\hline Private university hospital & $312(69.18)$ & $4.59 \pm 0.71$ & & & \\
\hline National \& public hospital & $25(5.54)$ & $4.63 \pm 0.74$ & & & \\
\hline Private hospital & $26(5.76)$ & $4.51 \pm 0.84$ & & & \\
\hline Specilization & & & & 1.436 & 0.239 \\
\hline Brain & $285(63.19)$ & $4.58 \pm 0.74$ & & & \\
\hline Spine & $110(24.39)$ & $4.57 \pm 0.68$ & & & \\
\hline Brain \& spine & $56(12.42)$ & $4.75 \pm 0.76$ & & & \\
\hline The number of residents & & & $e>b$ & 4.279 & 0.002 \\
\hline 1 (a) & $45(9.98)$ & $4.68 \pm 0.67$ & & & \\
\hline 2 (b) & $43(9.53)$ & $4.34 \pm 0.77$ & & & \\
\hline $3(c)$ & $85(18.85)$ & $4.65 \pm 0.76$ & & & \\
\hline $4(d)$ & $128(28.38)$ & $4.47 \pm 0.72$ & & & \\
\hline Over 5 (e) & $150(33.26)$ & $4.74 \pm 0.69$ & & & \\
\hline
\end{tabular}


Burnout of Korean Neurosurgeons I Kim TG

Table 5. Contiuned

\begin{tabular}{|c|c|c|c|c|c|}
\hline & Value & Mean \pm SD & Post hoc & $t / F$ & $p$-value \\
\hline Average working hours per day on weekdays & & & $d, c>b>a$ & 35.854 & $<0.001$ \\
\hline 8 to below 10 hours (a) & $72(15.96)$ & $4.04 \pm 0.60$ & & & \\
\hline 10 to below 12 hours (b) & $163(36.14)$ & $4.47 \pm 0.64$ & & & \\
\hline 12 to below 16 hours (c) & $174(38.58)$ & $4.87 \pm 0.65$ & & & \\
\hline Over 16 hours $(d)$ & $42(9.31)$ & $4.97 \pm 0.84$ & & & \\
\hline Working days on weekends per month & & & $e, d, c, b>a$ & 10.682 & $<0.001$ \\
\hline None (a) & $27(5.99)$ & $3.94 \pm 0.67$ & & & \\
\hline 1 (b) & $90(19.96)$ & $4.46 \pm 0.69$ & & & \\
\hline 2 (c) & $132(29.27)$ & $4.57 \pm 0.67$ & & & \\
\hline $3(d)$ & $76(16.85)$ & $4.69 \pm 0.73$ & & & \\
\hline Over 4 (e) & $126(27.94)$ & $4.82 \pm 0.72$ & & & \\
\hline Vacation per year & & & $a>e$ & 4.617 & 0.001 \\
\hline$<5$ days (a) & $92(20.40)$ & $4.72 \pm 0.76$ & & & \\
\hline $6-10$ days (b) & $258(57.21)$ & $4.66 \pm 0.70$ & & & \\
\hline $11-15$ days (c) & $84(18.63)$ & $4.40 \pm 0.71$ & & & \\
\hline $16-20$ days $(\mathrm{d})$ & $9(2.00)$ & $4.18 \pm 0.71$ & & & \\
\hline$>21$ days (e) & $7(1.55)$ & $4.03 \pm 0.88$ & & & \\
\hline Calls from interns or nurses & & & $d>a$ & 5.478 & $<0.001$ \\
\hline None (a) & $105(23.28)$ & $4.37 \pm 0.74$ & & & \\
\hline 1 (b) & $77(17.07)$ & $4.48 \pm 0.60$ & & & \\
\hline 2 (c) & $58(12.86)$ & $4.66 \pm 0.72$ & & & \\
\hline $3(d)$ & $30(6.65)$ & $4.84 \pm 0.79$ & & & \\
\hline 4 (e) & $43(9.53)$ & $4.54 \pm 0.72$ & & & \\
\hline Over 5 (f) & $138(30.60)$ & $4.79 \pm 0.71$ & & & \\
\hline Surgery without residents & & & $f>a$ & 6.691 & $<0.001$ \\
\hline None (a) & $194(43.02)$ & $4.41 \pm 0.71$ & & & \\
\hline $1(b)$ & $51(11.31)$ & $4.74 \pm 0.75$ & & & \\
\hline 2 (c) & $52(11.53)$ & $4.62 \pm 0.67$ & & & \\
\hline $3(d)$ & $30(6.65)$ & $4.72 \pm 0.52$ & & & \\
\hline 4 (e) & $44(9.76)$ & $4.59 \pm 0.77$ & & & \\
\hline Over $5(f)$ & $80(17.74)$ & $4.92 \pm 0.70$ & & & \\
\hline Rounds without residents & & & $f>a$ & 3.984 & 0.002 \\
\hline None (a) & $112(24.83)$ & $4.41 \pm 0.70$ & & & \\
\hline 1 (b) & $118(26.16)$ & $4.52 \pm 0.68$ & & & \\
\hline 2 (c) & $68(15.08)$ & $4.71 \pm 0.69$ & & & \\
\hline $3(d)$ & $54(11.97)$ & $4.66 \pm 0.71$ & & & \\
\hline 4 (e) & $34(7.54)$ & $4.76 \pm 0.75$ & & & \\
\hline Over 5 (f) & $65(14.41)$ & $4.83 \pm 0.80$ & & & \\
\hline Working as usual after overnight surgery & & & $\begin{array}{l}e>c, b \\
d, c>a\end{array}$ & 14.91 & $<0.001$ \\
\hline None (a) & $103(22.84)$ & $4.24 \pm 0.66$ & & & \\
\hline
\end{tabular}


Table 5. Contiuned

\begin{tabular}{|c|c|c|c|c|c|}
\hline & Value & Mean \pm SD & Post hoc & $t / F$ & $p$-value \\
\hline $1(b)$ & $148(32.82)$ & $4.54 \pm 0.70$ & & & \\
\hline 2 (c) & $101(22.39)$ & $4.72 \pm 0.68$ & & & \\
\hline $3(d)$ & $61(13.53)$ & $4.85 \pm 0.65$ & & & \\
\hline Over 4 (e) & $38(8.43)$ & $5.09 \pm 0.80$ & & & \\
\hline The level of job stress & & & $\begin{array}{c}e>c>a \\
d>b\end{array}$ & 64.358 & $<0.001$ \\
\hline Not at all (a) & $5(1.11)$ & $3.40 \pm 0.33$ & & & \\
\hline Not (b) & $15(3.33)$ & $3.66 \pm 0.56$ & & & \\
\hline Normal (c) & $104(23.06)$ & $4.12 \pm 0.56$ & & & \\
\hline Yes (d) & $178(39.47)$ & $4.55 \pm 0.56$ & & & \\
\hline Very yes (e) & $149(33.04)$ & $5.13 \pm 0.63$ & & & \\
\hline Satisfaction with salary level & & & $a>c$ & 7.707 & $<0.001$ \\
\hline Not at all (a) & $179(39.69)$ & $4.83 \pm 0.74$ & & & \\
\hline Not (b) & $116(25.72)$ & $4.47 \pm 0.66$ & & & \\
\hline Normal (c) & $108(23.95)$ & $4.41 \pm 0.67$ & & & \\
\hline Yes (d) & $37(8.20)$ & $4.51 \pm 0.75$ & & & \\
\hline Very yes (e) & $11(2.44)$ & $4.44 \pm 0.78$ & & & \\
\hline Satisfaction with welfare system & & & $a>d$ & 3.707 & 0.006 \\
\hline Not at all (a) & $144(31.93)$ & $4.78 \pm 0.73$ & & & \\
\hline Not (b) & $139(30.82)$ & $4.54 \pm 0.73$ & & & \\
\hline Normal (c) & 113 (25.06) & $4.49 \pm 0.71$ & & & \\
\hline Yes (d) & $40(8.87)$ & $4.44 \pm 0.66$ & & & \\
\hline Very yes (e) & $15(3.33)$ & $4.61 \pm 0.67$ & & & \\
\hline Satisfaction with human relationships & & & $a, b>d, e$ & 5.935 & $<0.001$ \\
\hline Not at all (a) & $26(5.76)$ & $5.00 \pm 0.92$ & & & \\
\hline Not (b) & $49(10.86)$ & $4.95 \pm 0.75$ & & & \\
\hline Normal (c) & $173(38.36)$ & $4.60 \pm 0.75$ & & & \\
\hline Yes (d) & $165(36.59)$ & $4.48 \pm 0.61$ & & & \\
\hline Very yes (e) & $38(8.43)$ & $4.41 \pm 0.66$ & & & \\
\hline
\end{tabular}

Values are presented as number (\%). SD : standard deviation

year; frequent calls from interns or nurses, surgeries without residents, or rounds without residents; or frequent days of working-as-usual after overnight surgery, a high level of job stress, low-level satisfaction with salary level, low-level satisfaction with welfare, or low-level satisfaction with human relationships experienced a high level of burnout. Position, hospital location, hospital size, hospital type, and specialization were not related to burnout.

\section{Linear correlation analysis}

Pearson's correlation analyses were conducted to explore the relationships between the independent variables and dependent variables (Table 6). The correlation between the independent variables were : work intensity and work-life balance was $\mathrm{r}=0.555(p<0.001)$, work intensity and work-life balance-family and leisure was $r=0.586(p<0.001)$, and work intensity and work-life balance-growth were $r=0.409(p<0.001)$. In the relationship between the independent variables and dependent variables, statistically significant, positive correlations were found : 
Table 6. Correlation analysis

\begin{tabular}{lcccc} 
& a & b & c & d \\
\hline a. Work intensity & 1 & & & \\
b. Work-life balance & $0.555^{*}$ & 1 & & \\
c. Work-life balance-family \& leisure & $0.586^{*}$ & $0.951^{*}$ & 1 & 1 \\
d. Work-life balance-growth & $0.409^{*}$ & $0.892^{*}$ & $0.709^{*}$ & $0.597^{*}$ \\
e. Burnout & $0.620^{*}$ & $0.693^{*}$ & $0.674^{*}$ & 1 \\
\hline${ }^{*} p<001$ & & & &
\end{tabular}

${ }^{*} p<0.001$

Table 7. The effect of work intensity and work-life balance on burnout

\begin{tabular}{|c|c|c|c|c|c|c|c|c|c|c|c|c|}
\hline & \multicolumn{4}{|c|}{ Model 1} & \multicolumn{4}{|c|}{ Model 2} & \multicolumn{4}{|c|}{ Model 3} \\
\hline & B & $\beta$ & $\mathrm{t}$ & $p$-value & B & $\beta$ & $\mathrm{t}$ & $p$-value & B & $\beta$ & $\mathrm{t}$ & $p$-value \\
\hline (Constant) & 3.391 & & 16.474 & $<0.001$ & 2.886 & & 12.546 & $<0.001$ & 1.581 & & 7.059 & $<0.001$ \\
\hline Gender, female & 0.285 & 0.085 & 2.004 & 0.046 & 0.239 & 0.071 & 1.932 & 0.054 & 0.137 & 0.041 & 1.280 & 0.201 \\
\hline Age (years) & 0.026 & 0.030 & 0.676 & 0.499 & 0.006 & 0.007 & 0.173 & 0.862 & 0.023 & 0.027 & 0.808 & 0.419 \\
\hline The number of residents & 0.033 & 0.059 & 1.304 & 0.193 & 0.021 & 0.037 & 0.944 & 0.346 & 0.003 & 0.005 & 0.143 & 0.886 \\
\hline $\begin{array}{l}\text { Average working hours per day } \\
\text { on weekdays }\end{array}$ & 0.243 & 0.290 & 6.000 & $<0.001$ & 0.131 & 0.156 & 3.556 & $<0.001$ & 0.020 & 0.024 & 0.588 & 0.557 \\
\hline $\begin{array}{l}\text { Working days on weekends } \\
\text { per month }\end{array}$ & 0.015 & 0.027 & 0.551 & 0.582 & -0.011 & -0.018 & -0.440 & 0.660 & -0.030 & -0.052 & -1.441 & 0.150 \\
\hline Vacation per year & -0.017 & -0.093 & -2.112 & 0.035 & -0.010 & -0.053 & -1.391 & 0.165 & -0.002 & -0.014 & -0.411 & 0.681 \\
\hline Calls from interns or nurses & 0.031 & 0.087 & 1.849 & 0.065 & 0.024 & 0.065 & 1.611 & 0.108 & 0.018 & 0.048 & 1.381 & 0.168 \\
\hline Surgery without residents & 0.032 & 0.086 & 1.849 & 0.065 & 0.022 & 0.059 & 1.450 & 0.148 & 0.010 & 0.026 & 0.736 & 0.462 \\
\hline Rounds without residents & 0.036 & 0.084 & 1.846 & 0.066 & 0.023 & 0.054 & 1.352 & 0.177 & 0.024 & 0.056 & 1.635 & 0.103 \\
\hline $\begin{array}{l}\text { Working as usual after } \\
\text { overnight surgery }\end{array}$ & 0.088 & 0.148 & 3.076 & 0.002 & 0.053 & 0.089 & 2.077 & 0.038 & 0.015 & 0.025 & 0.683 & 0.495 \\
\hline The level of job stress & & & & & 0.362 & 0.444 & 10.751 & $<0.001$ & 0.133 & 0.164 & 3.887 & $<0.001$ \\
\hline Satisfaction with salary level & & & & & -0.035 & -0.052 & -1.128 & 0.260 & -0.020 & -0.029 & -0.727 & 0.467 \\
\hline $\begin{array}{l}\text { Satisfaction with welfare } \\
\text { system }\end{array}$ & & & & & 0.011 & 0.017 & 0.369 & 0.712 & 0.015 & 0.022 & 0.546 & 0.585 \\
\hline $\begin{array}{l}\text { Satisfaction with human } \\
\text { relationships }\end{array}$ & & & & & -0.098 & -0.131 & -3.479 & 0.001 & -0.069 & -0.092 & -2.770 & 0.006 \\
\hline Work intensity & & & & & & & & & 0.314 & 0.303 & 7.154 & $<0.001$ \\
\hline $\begin{array}{l}\text { Work-life balance-family \& } \\
\text { leisure }\end{array}$ & & & & & & & & & 0.216 & 0.223 & 4.173 & $<0.001$ \\
\hline Work-life balance-growth & & & & & & & & & 0.147 & 0.173 & 3.779 & $<0.001$ \\
\hline $\operatorname{adj} R 2(\triangle \operatorname{adj} R 2)$ & \multicolumn{4}{|c|}{0.245} & \multicolumn{4}{|c|}{$0.436(0.192)$} & \multicolumn{4}{|c|}{$0.583(0.145)$} \\
\hline$F(p)$ & \multicolumn{4}{|c|}{$15.570(0.000)$} & \multicolumn{4}{|c|}{$25.835(0.000)$} & \multicolumn{4}{|c|}{$37.952(0.000)$} \\
\hline Durbin-Watson's d(du) & \multicolumn{4}{|c|}{$1.967(1.891)$} & \multicolumn{4}{|c|}{$1.931(1.914)$} & \multicolumn{4}{|c|}{$2.056(1.924)$} \\
\hline K-S (p) & \multicolumn{4}{|c|}{$0.040(0.082)$} & \multicolumn{4}{|c|}{$0.035(0.200)$} & \multicolumn{4}{|c|}{$0.029(0.200)$} \\
\hline$x^{2}$ & \multicolumn{4}{|c|}{$10.800(0.373)$} & \multicolumn{4}{|c|}{$13.771(0.467)$} & \multicolumn{4}{|c|}{$17.678(0.409)$} \\
\hline
\end{tabular}

$\mathrm{d}\left(\mathrm{d}_{\mathrm{u}}\right)$ : Durbin-Watson coefficient (Durbin-Watson's upper bound), K-S : Kolmogorov-Smirnov's normality test, $X^{2}$ : Breusch-Pagan's equal variance test 
work intensity and burnout $\mathrm{r}=0.620(p<0.001)$, and work-life balance and burnout $\mathrm{r}=0.693$ ( $p<0.001)$; work-life balancefamily and leisure and burnout $\mathrm{r}=0.674(p<0.001)$, and worklife balance-growth and burnout $\mathrm{r}=0.597(p<0.001)$.

\section{Regression analysis}

Regression analysis was conducted (Table 7) using variables that were significantly related in the analysis of burnout and the socio-demographic characteristics (Table 5) and in the correlation analysis (Table 6). The socio-demographic variables significantly related to burnout included gender, age, number of residents, average working hours per day on weekdays, working days on weekends per month, vacation per year, calls from interns or nurses, surgery without residents, rounds without residents, working-as-usual after overnight surgery, level of job stress, satisfaction with the salary level, satisfaction with the welfare system, and satisfaction with human relationships. The significant independent variables in the correlation analysis were work intensity, work-life balance-family and leisure and work-life balance-growth. The effects of work intensity, work-life balance-family and leisure, and work-life balance-growth on burnout were analyzed by hierarchical regression analysis with primary and secondary control of variables. The primary control variables were gender, age, number of residents, average working hours per day on weekdays, working days on weekends per month, vacation days per year, calls from interns or nurses, surgery without residents, rounds without residents, and working-as-usual after overnight surgery (that is, socio-demographic characteristics and objective work environment). The secondary control variables were the level of job stress, satisfaction with salary level, satisfaction with the welfare system, and satisfaction with human relationships (that is, subjective work environment).

In order to conduct regression analysis, the autocorrelation of the dependent variables and the multicollinearity between the independent variables were examined. The autocorrelation of the dependent variables is based on the Durbin-Watson index; if the Durbin-Watson index is close to $2(\mathrm{dU}<\mathrm{d}<4-\mathrm{dU})$, the dependent variables are dependent without autocorrelations ${ }^{9}$. The multiple collinearity between the independent variables is determined to be non-multicollinear if the variance inflation factor (VIF) index appears to be less than 10. To determine the goodness-of-fit of the model, the residual test was performed using the Kolmogorov-Smirnov normality test and Breusch-
Pagan's equal variance test.

The Durbin-Watson index of the dependent variable was $1.967(\mathrm{dU}=1.891<\mathrm{d})$ in the first stage model that controlled for the first control variables such as gender, age, the number of residents, average working hours per day on weekdays, working days on weekends per month, vacation days per year, calls from interns or nurses, surgery without residents, rounds without residents, and working-as-usual after overnight surgery. The VIF index was less than 10, which was not multicollinear. Therefore, it was appropriate to conduct a regression analysis. The results of the regression analysis showed that gender ( $p=0.046$ ), average working hours per day on weekdays $(p<0.001)$, vacation days per year $(p=0.035)$, and working-asusual after overnight surgery $(p=0.002)$ had significant effects of burnout. Furthermore, female respondents $(\beta=0.285)$, respondents with more average working hours per day on weekdays $(\beta=0.243)$, fewer vacation days per year $(\beta=-0.017)$, and frequent days of working-as-usual after overnight surgery ( $\beta=0.088$ ), showed higher levels of burnout; $24.5 \%(p<0.05)$ of the variance was explained. As a result of the goodness-of-fit test of the model containing only primary control variables, Model 1 was found suitable for the normality $(p=0.082)$ and equal variance $(p=0.373)$ tests of the standardized residuals.

The Durbin-Watson index of the dependent variable, burnout, was $1.931(\mathrm{dU}=1.914<\mathrm{d})$ in the second-stage model that controlled for the second control variables, such as the level of job stress, satisfaction with salary level, satisfaction with the welfare system, and satisfaction with human relationships. The VIF index was less than 10, which was not multicollinear; therefore, it was appropriate to perform a regression analysis. The results of the regression analysis showed that the level of job stress $(p<0.001)$ and satisfaction with human relationships ( $p=0.001$ ) had significant effects on burnout. In the case of high level of job stress $(\beta=0.362)$ and low level of satisfaction with human relationships ( $\beta=-0.098)$, indicating a higher level of burnout; $19.2 \%(p<0.05)$ of the variance was explained. As a result of the goodness-of-fit test of this model, which contained secondary control variables, Model 2 was found suitable for the normality $(p=0.200)$ and equal variance $(p=0.467)$ tests of the standardized residuals.

The Durbin-Watson index of the burnout was 2.056 ( $\mathrm{dU}=1.924<\mathrm{d}$ ) in the third stage model that included the independent variables such as work intensity, work-life balancefamily and leisure, and work-life balance-growth. The VIF in- 
dex was less than 10 , which was not multicollinear; therefore, it was appropriate to perform a regression analysis. The results of the regression analysis showed that work intensity $(p<0.001)$, work-life balance-family and leisure $(p<0.001)$, and work-life balance-growth $(p<0.001)$ had significant effects on burnout. In the case of high work intensity $(\beta=0.314)$, negative work-life balance-family and leisure $(\beta=0.216)$, and negative work-life balance-growth $(\beta=0.147)$, the results were a high level of burnout; $14.5 \%(p<0.05)$ of the variance was explained. In Model 3, the explanatory power increased significantly by $14.5 \%(p<0.05)$; the independent variables including work intensity, work-life balance-family and leisure, and work-life balance-growth had significant effects on burnout. As a result of the goodness-of-fit test of the model containing independent variables, Model 3 was found suitable for the normality ( $p=0.200)$ and equal variance $(p=0.409)$ tests of the standardized residuals.

\section{DISCUSSION}

The Special Act on Korean Medical Residents enacted in 2015 is a law intended to improve the working environment of the residents by setting a practical upper limit of working hours by allowing the residents' an average of 80 hours working hours per week and adding 8 hours per week for educational purposes only. In the case of training hospitals that violate this law, there are enforcement provisions that can impose corrective orders and penalties by the Ministry of Health and Welfare. However, a lot of controversy surrounded the Special Act on Korean Medical Residents, such as the deficiency of the training time, the lack of training continuity, or the manpower gap. In this regard, the Korean Hospital Association argued that if the training time of the resident is reduced, the training hospital may need to hire about 3600 more doctors, and the cost of labor needed may be from about KRW 470 million to KRW 2.75 billion per training hospital. In response, the Ministry of Health and Welfare proposed the support of cost of labor through the hospitalist system as an alternative; however, the hospitalist system has not yet been settled, and the manpower gap that was a concern is still a challenge in the field. Hospitals have found that it is difficult to recruit the number of specialists or hospitalists necessary because the low payment of the health insurance systems is not cost-preserving, making it difficult to generate medical profits through medical treatment. Therefore, existing manpower such as fellows or professors will be responsible for the vacancy, not the alternative, such as recruitment of manpower, which will inevitably lead to side effects of the increased workload.

Despite these concerns from stakeholders, such as lack of medical care vacancies, manpower expansion plans, and increased labor costs, after the enactment of the law, the Special Act on Korean Medical Residents was fully implemented on December 23, 2017. The effect of the new law or system should be evaluated in a broad manner for the appropriate period. Considering that one of the important circumstances in which the Special Act on Korean Medical Residents was enacted is patient safety, it is necessary to examine the work intensity, work-life balance, and burnout level of neurosurgeons working in training hospitals because these variables are associated with patient safety. In particular, the increase in the level of burnout experienced by neurosurgeons who perform neurosurgery is directly related to patient safety.

The term burnout was introduced and used by American psychoanalyst Herbert Freudenberger ${ }^{1)}$. He used the term burnout, describing the frustrations and psychological problems of clinicians and psychologists who volunteered at free clinics for drug addicts. After that, social psychologists Maslach and Jackson defined EE as being due to chronic interpersonal relationships and stress in work, DP, and reduced personal accomplishment as three factors of burnout ${ }^{10)}$. Later, Shirom and Melamed, unlike Maslach, identified physical fatigue and cognitive fatigue as important factors of burnout instead of DP and reduced personal accomplishment. These burnout can cause occupational problems such as productivity decline, job dissatisfaction, and turnover, and are known to be associated with various physical (diabetes, dyslipidemia, metabolic syndrome, coronary artery disease, muscle pain, headache, chronic fatigue, accident and early death) and mental (depression, sleep disorder, and mental disorder) health problems ${ }^{17)}$. Burnout has been studied in relation to EE of health professions such as doctors and nurses. It is known that the frequency and prevalence is relatively high in medical staff. In a meta-analytic study that compiled studies from 45 countries from 1991 to 2018, about 67\% of doctors reported experiencing burnout ${ }^{16)}$. Medical staff experience high levels of stress in the process of treating patients. It has been reported that the burnout in medical staffs is related to an increase 
in patient safety accidents, a decrease in patient satisfaction, and a decrease in medical quality ${ }^{13}$.

Of course, it is impossible to compare the situation before and after the law enters into force with only one survey. Therefore, this study aimed to identify the work intensity, work-life balance, and burnout of neurosurgeons in training hospitals after the enforcement of this law and aimed to collect opinions (about this law) from neurosurgeons who have experienced the enforcement of this law. In this study, the work environment of neurosurgeons, such as fellows who were in charge of work vacancies in training hospitals after the Special Act on Korean Medical Residents, was confirmed, and their work intensity, work-life balance, and burnout were investigated to identify the relationship between them.

This study sample is highly representative with $51 \%$ of the specialists in the training hospitals responding. While the percentage of female respondents (4.9\%) is higher than the ratio of the total neurosurgery specialists (about $1.8 \%=59 / 3329$, as of March 2020) and, according to statistics from December 2018 when 32 female neurosurgeons were working at training hospitals, the number of 22 responses by female neurosurgeons in this study is presumed to be higher than the proportion of male respondents in the training hospitals ${ }^{6}$.

The groups with the largest number of respondents were males, 40-49 years of age. Based on objective work environment, the largest number of respondents were professors, worked in hospitals in Seoul, hospitals with 800-999 beds, private university hospitals and had a specialization in the brain, worked with five or more residents, averaged 12-16 working hours per day on weekdays, two working days on weekends per month, vacations per year with 6-10 days, five or more calls from interns or nurses, surgery without residents zero times a month, rounds without residents once a month, days working-as-usual after overnight surgery once a month. Regarding their subjective work environment, the largest number of respondents indicated on a 5-point scale, their level of job stress as 4 points, satisfaction with salary level 1 point, satisfaction with welfare system 1 point, and satisfaction with human relationships 3 points.

These may be the approximate status and perceptions of neurosurgeons working at the training hospital in Korea. What is surprising about these status and perceptions is that, if the average working hours per day on weekdays are 12-16 hours and the average working days on weekends per month is 2 days, there is no difference from the residents who work 80 hours per week. Alternatively, they work relatively longer. Once a month, they have to work as-usual after overnight surgery and the average number of vacation days per year is very small, with 6-10 days; their level of job stress is very high, with 4 out of 5 points, and they were found to suffer from significant physical and mental hardships. The cause of these situations seems to have been around for a long time, not solely the Special Act on Korean Medical Residents. However, it seems certain that the Special Act on Korean Medical Residents has exacerbated the situation; for this purpose, we included questions specific to the Special Act. According to these questions, there were many respondents reported that there was a shortage of time (70.5\%) due to the need to replace the work of the residents, and that this lack of time interfered with the original work of patient care (59.6\%) and their academic research activities (84.9\%). In other words, the Special Act on Korean Medical Residents has a negative impact on the work conditions of the neurosurgeons and interferes with their original work such as patient care and research. In addition, patient satisfaction was reduced by these situations (61.6\%), and as an alternative, medical assistants such as physician assistants were absolutely needed $(88.5 \%)^{3)}$. In this response, the following facts can be estimated. The implementation of the Special Act on Korean Medical Residents without alternatives would have been a very challenging situation for neurosurgeons in training hospitals who had been suffering from heavy burdens, and most of them are claiming the necessity of medical assistant manpower.

These facts are seen in the other questions in our study. According to the results of our study, the work intensity was very high at 3.95 and work-life balance was also very high at 3.57, when measured on a 5-point scale. The burnout was also very high at 4.60, on a 7-point scale. In particular, many researchers use the MBI-Human Services Survey 22 questions on a 7-point scale in relation to burnout, which is usually seen as a case of meeting $\mathrm{EE} \geq 27, \mathrm{DP} \geq 10$, and $\mathrm{PA} \leq 33$ as a definition of burnout $^{2,11,14,15,18)}$. In this study, 280 (62.1\%) of 451 respondents were found to be in a state of burnout, a very high level that can be seen in two out of three neurosurgeons in the training hospitals. Therefore, it is believed that neurosurgeons in training hospitals are now in a very dangerous condition.

When the associate professors under 60, working in large hospitals with more than five residents, work long days with 
short holidays, the work intensity is high-a very natural result. The work intensity was naturally high in the case of those performing many surgeries without residents, working many days as-usual after overnight surgery, experiencing high levels of job stress, and having high salary satisfaction. Welfare satisfaction, gender, hospital location, hospital type, specialization, number of rounds without residents, and satisfaction with human relationships were not related to work intensity. Through this, it was confirmed that the workplace with a lot of salary has a lot of work. And welfare, and human relationships has no correlation with work intensity; similarly, the location, types of training hospitals, and gender of the respondent have no impact on work intensity. In the case of receiving three calls from nonresidents a month, the work intensity was higher than in the case of receiving four calls. This result is not consistent with the Scheffe post hoc analysis, so it is difficult to interpret.

The effect of the socio-demographic characteristics on work-life balance was similar to the general expectations, such as work intensity. However, the difference in the impact on work intensity was that the work-life balance of neurosurgeons with specializations in both brain and spine and that of female respondents was worse, and the hospital size was irrelevant. The effect of sociodemographic characteristics on burnout was similar to the general expectation, as did work intensity and work-life balance. However, while female respondents were more likely to experience burnout, their positions, hospital size, and specialization were irrelevant. In other words, even though the work intensity of females and the work intensity of neurosurgeons with specializations in brain and spine were not higher, work-life balance was less; burnout was worse in females. There was no significant difference in stress level based on specialization ( $p=0.083$, not described separately), so work intensity and stress level cannot explain the lower level of work-life balance experienced by neurosurgeon with specializations in brain and spine. There was no significant difference in stress level by gender $(p=0.463$, not described separately), so work intensity and stress level cannot explain the lower work-life balance and high level of burnout of females. These two factors are beyond the scope of this study, so additional research on these topics should be undertaken in the future.

The result of the correlation analysis, showed strong positive correlation between all independent variables such as work intensity ( $\mathrm{r}=0.620)$, work-life balance-family and leisure $(r=0.693)$, and work-life balance-growth $(r=0.597)$ with the dependent variable, burnout. In other words, if the work intensity is high and the work-life balance is poor, the burnout becomes more intense, which can be seen as consistent with the general prediction. Using the variables as dependent variables that showed significant results in the correlation analysis and the variables that showed significant results in the analysis of burnout, based on to the socio-demographic characteristics already implemented, the hierarchical regression analysis was conducted with burnout as the dependent variable. By controlling the socio-demographic characteristics including objective work environment and subjective work environment, the effects of work intensity and work-life balance on burnout (model 3) were analyzed. Work intensity $(\mathrm{B}=0.314)$, work-life balance-family and leisure $(B=0.216)$, work-life balancegrowth $(B=0.147)$, job stress $(B=0.133)$, and satisfaction with human relationships $(B=-0.069)$ were shown to be significant, and to affect burnout in the order above. The overall explanatory power was $58.3 \%(p<0.05)$, and the explanatory power of model 3 with the addition of independent variables such as work intensity and work-life balance was $14.5 \%(p<0.05)$. In other words, it was found that work intensity, work-life balance-family and leisure, work-life balance-growth, job stress, and satisfaction with human relationships in the workplace had significant effects on burnout in this order.

\section{CONCLUSION}

This study showed that Korean neurosurgeons working at training hospitals had a high level of work intensity and job stress and a low level of work-life balance. In addition, 280 (62.1\%) of 451 neurosurgeons were found to experience burnout related to factors such as work intensity, work-life balance, job stress, and satisfaction with human relationships. In particular, these factors seem to have deteriorated further after the implementation of the Special Act on Korean Medical Residents. These significantly high levels of burnout among Korean neurosurgeons who care for patients with both brain and spinal diseases may have a critical impact on the health of Korean patients. Therefore, it is recommended that the Korean Neurosurgical Society and the Korean Government make efforts to improve the factors that affect burnout among Ko- 
rean neurosurgeons.

\section{CONFLICTS OF INTEREST}

No potential conflict of interest relevant to this article was reported.

\section{INFORMED CONSENT}

This type of study does not require informed consent.

\section{AUTHOR CONTRIBUTIONS}

\author{
Conceptualization : TGK \\ Data curation : TGK \\ Formal analysis : TGK \\ Funding acquisition : TGK \\ Methodology : TGK \\ Project administration : TGK \\ Visualization : TGK \\ Writing - original draft : TGK \\ Writing - review \& editing : TGK
}

\section{ORCID}

Tae Gon Kim https://orcid.org/0000-0001-6258-6412

\section{- Acknowledgements}

This study was supported by a grant from the Policy Research Funds of the Korean Neurosurgical Society.

\section{References}

1. Ahola K, Hakanen J : Job strain, burnout, and depressive symptoms: a prospective study among dentists. J Affect Disord 104 : 103-110, 2007
2. Busis NA, Shanafelt TD, Keran CM, Levin $K H$, Schwarz HB, Molano JR, et al. : Burnout, career satisfaction, and well-being among US neurologists in 2016. Neurology $88: 797-808,2017$

3. Cho KG : Role and effectiveness of nurse practitioner in neurosurgical field. J Korean Neurosurg Soc 25 : 1377-1382, 1996

4. Choi HY, Chung NW : Perfectionism tendency, social support, and burnout among counselors. Korean J Health Psychol 8 : 279-300, 2003

5. Janssen $0:$ Job demands, perceptions of effort-reward fairness and innovative work behaviour. J Occup Organ Psychol 73 : 287-302, 2000

6. Jung TY, Kim EY, Park MS : Herstory of the Korean Women Neurosurgical Society since 2008. J Korean Neurosurg Soc 62 : 619-625, 2019

7. Kim JW, Park CY, Son YM, Jang H : The conceptual understanding on 'work-life balance' and its effectiveness. Journal of Leisure Studies $2:$ :29-48, 2005

8. Kim JW, Park JY : A study on the development of a 'work-life balance' scale. Journal of Leisure Studies 5 : 53-69, 2008

9. Lee IH : Easy Flow Regression Analysis. Seoul : Hannarae, 2014

10. Maslach C, Jackson SE : The measurement of experienced burnout. J Organ Behav 2 : 99-113, 1981

11. Maticorena-Quevedo J, Beas R, Anduaga-Beramendi A, Mayta-Tristán $P$ : Prevalence of burnout syndrome in peruvian physicians and nurses, ENSUSALUD 2014. Rev Peru Med Exp Salud Publica 33 : 241-247, 2016

12. Nunnally JC : Psychometric Theory, ed 2. New York : McGraw-Hill, 1978

13. Panagioti M, Geraghty K, Johnson J, Zhou A, Panagopoulou E, ChewGraham $C$, et al. : Association between physician burnout and patient safety, professionalism, and patient satisfaction: a systematic review and meta-analysis. JAMA Intern Med 178 : 1317-1331, 2018

14. Pedersen $A F$, Ingeman $M L$, Vedsted $P$ : Empathy, burn-out and the use of gut feeling: a cross-sectional survey of Danish general practitioners. BMJ Open 8 : e020007, 2018

15. Pedersen AF, Sørensen JK, Bruun NH, Christensen B, Vedsted P : Risky alcohol use in Danish physicians: associated with alexithymia and burnout? Drug Alcohol Depend 160 : 119-126, 2016

16. Rotenstein LS, Torre M, Ramos MA, Rosales RC, Guille C, Sen S, et al. : Prevalence of burnout among physicians: a systematic review. JAMA 320 : 1131-1150, 2018

17. Salvagioni DAJ, Melanda FN, Mesas AE, González AD, Gabani FL, Andrade SM : Physical, psychological and occupational consequences of job burnout: a systematic review of prospective studies. PLoS One 12 : e0185781, 2017

18. Shanafelt TD, Hasan O, Dyrbye LN, Sinsky C, Satele D, Sloan J, et al. : Changes in burnout and satisfaction with work-life balance in physicians and the general US working population between 2011 and 2014. Mayo Clin Proc 90 : 1600-1613, 2015 